\title{
Strengthening Quadriceps Muscles with Neuromuscular Electrical Stimulation Following Total Hip Replacement: a Review
}

\author{
Louise C. Burgess $^{1} \cdot \operatorname{lan}$ D Swain ${ }^{1} \cdot$ Paul Taylor $^{2,3,4} \cdot$ Thomas W. Wainwright $^{1}$
}

Published online: 9 May 2019

(C) The Author(s) 2019

\begin{abstract}
Purpose of Review Functional recovery from total hip replacement can be suboptimal and deficits in quadriceps muscle strength may hinder the return to activities of daily living. Neuromuscular electrical stimulation (NMES) devices have long been used to preserve and restore skeletal muscle mass and function following periods of muscle atrophy due to immobilisation. Here, we evaluate the potential role of NMES for strengthening the quadriceps muscles following hip replacement.

Recent Findings Two studies have investigated the effects of NMES on quadriceps strength following hip replacement. NMES in addition to exercise training is reported to reduce length of stay and improve gait speed, stair climbing performance and sit-tostand scores in one study. Conversely, the other study reports no significant effect of NMES on length of stay or gait speed, but instead, benefits to knee extensor strength of the operated side, functional status and independence. The benefits of NMES for the treatment of atrophic musculature following knee replacement are better established.

Summary Although it is not possible to offer best-practice recommendations for clinical rehabilitation, the promising approach of NMES following total hip replacement requires further investigation. When used as an adjunctive treatment to standard care physiotherapy, NMES may facilitate recovery and, when used immediately post-surgery, can enable a high exercise volume, with little effort, at a time point where muscle inhibition and atrophy are most prevalent.
\end{abstract}

Keywords Neuromuscular electrical stimulation (NMES) $\cdot$ Total hip replacement $\cdot$ Rehabilitation $\cdot$ Physiotherapy $\cdot$ Functional recovery

\section{Introduction}

For some time, total hip replacement has been recognised as a clinically successful and cost-effective surgical proce-

This article is part of the Topical Collection on Musculoskeletal Rehabilitation

Louise C. Burgess

lburgess@bournemouth.ac.uk

1 Orthopaedic Research Institute, Bournemouth University, Executive Business Centre, 89 Holdenhurst Road, Bournemouth BH8 8EB, UK

2 Department Clinical Science and Engineering, Salisbury District Hospital, Salisbury, Wiltshire SP2 8BJ, UK

3 Odstock Medical Limited, Salisbury District Hospital, Salisbury, Wiltshire SP2 8BJ, UK

4 Faculty of Health and Social Science, Bournemouth University, Royal London House, 109 Christchurch Road, Bournemouth BH1 3HT, UK dure. The operation has seen significant change over time and the technical development of prostheses and advances in surgical techniques have led to increasingly successful outcomes [1-3]. It has been acknowledged, however, that full recovery following surgery does not equate to being discharged early without complications [4]. Recovery is a complex process that is only complete once the patient returns to normal function [5]. This has been promoted more recently in Enhanced Recovery after Surgery programmes, where there is an additional emphasis on enabling a patient to return to work, activities of daily living and pre-surgery function [6]. There is evidence to suggest that physical activity levels do not increase after surgery [7], and in some cases, patients are less active at 2-year follow-up than before hospital admission [8•]. As return to normal function becomes the benchmark for recovery after major surgery, a focus of perioperative care should now be on post-discharge support and physical rehabilitation strategies [9•]. 


\section{Functional Recovery}

To improve functional recovery from hip replacement surgery, it is first important to identify which muscle groups are functionally relevant for the motor performance of activities of daily living. Lower extremity muscle mass has been reported as a key determinant of physical performance amongst mobility-limited older adults, and total leg mass and muscle strength are considered strong independent predictors of the level of functional impairment [10,11]. More specifically, there is evidence to suggest an association between quadriceps femoris strength and functional outcomes [12], as quadriceps muscle strength is functionally relevant for performance of daily tasks $[13,14]$. Both knee extensor concentric and eccentric strength and power are associated with improved performance of the sit-to-stand movement [15] and muscle activity that produces sufficient force is essential to enable a patient to walk, rise from a chair or climb stairs [16]. In addition, muscle weakness has been associated with slower gait speeds and an increased risk of falls [17].

\section{Challenges of Functional Recovery}

Unfortunately, many of the muscles most important for functional activity correlate with those negatively affected by hip osteoarthritis, the primary indication for a hip replacement [18] and by substantial strength decreases in the first week following hip replacement surgery. Existing literature suggests that unilateral hip osteoarthritis is characterised by generalised muscle weakness of the affected leg [19] and hip muscle strength and leg press power are reported to decrease substantially in the first week after surgery [20]. There is a consistent, strong evidence that reports a reduced quadriceps muscle size in the affected leg, compared with the contralateral leg, of an individual with osteoarthritis of the hip [19], and hip replacement patients are commonly affected by loss of quadriceps function and impairments that may remain for years following discharge [21]. Preoperative osteoarthritic pain can lead to reduced physical activity levels as patients suffer from pain-related immobilisation or reduced functionality [22], which subsequently leads to muscle disuse atrophy. Muscle weakness may also occur due to a reduction in the level of motor unit activation [19] and this deficit occurs when there is failure to recruit all available motor units or a reduction in maximal motor unit discharge rate from those motor units which are recruited [23].

\section{Electrical Stimulation Therapy}

As well as activation of muscles via the bodies' nervous system, muscles can be contracted by the application of an external electrical stimulation. Electrotherapy is the delivery of energy from a device that enters into a tissue and results in a change in one or more physiological events [24]. Neuromuscular electrical stimulation (NMES) involves the application of preprogrammed trains of stimuli to superficial skeletal muscles, by means of surface electrodes placed over the muscle belly, in an attempt to evoke visible, involuntary contractions [25]. NMES devices have long been used to either preserve [26] or restore [27] skeletal muscle mass and function following periods of muscle atrophy due to immobilisation. Despite the promising approach to rehabilitation using perioperative NMES, devices are not used in routine clinical practice following joint replacement. NMES can strengthen atrophic musculature weakened from disuse and orthopaedic patients, immobilised due to joint pain or by the fixation of casts and splints, may benefit from this treatment strategy [28].

Whilst it is thought that the force increases induced by NMES are similar, but not greater than those induced by voluntary training [29], it can be used in the acute recovery phase following surgery and may offer an adjunctive therapy or alternative treatment strategy for those confined to bed rest or unable to mobilise independently. Following the immediate postoperative phase, NMES combined with exercise interventions has been advocated as an optimal treatment strategy, as the adaptions evoked by NMES are not just confined to the activated muscle but also involve neural adaptions through reflex inputs to the spinal cord and supraspinal centres [30]. There is evidence that demonstrates a better functional recovery, particularly in the immediate phase, following total knee replacement for patients using NMES [31 •]; however, research in cohorts of hip replacement patients is limited and more studies are warranted [9॰]. Therefore, our review aims to summarise and evaluate the current evidence for stimulating the quadriceps muscles following hip replacement surgery, in order to improve functional recovery.

\section{Methodology}

A literature review was conducted to examine current published evidence that considers the use of NMES for strengthening the quadriceps muscles following hip replacement. A computer-based search was completed in March 2018, and the electronic database sourced included PubMed, Cochrane library, Google Scholar, Science Direct, PsychINFO, CINAHL Complete and Medline Complete. The search reviewed all fields of the available literature, published in the English language (or those where a translation was available) to the earliest record on file. The reference lists of articles, review papers and textbooks were also scanned for additional papers. Studies were considered eligible for inclusion within the synthesis if they met the specified inclusion and exclusion criteria listed in Table 3 in the Appendix. As there are two categories of NMES, functional electrical stimulation search terms were also included to capture studies where the transmission of an electrical impulse over the skin had been used to enhance functional activities in neurologically 
impaired individuals. Studies which used sensory-level electrical stimulation (namely transcutaneous electrical nerve stimulation) were excluded.

\section{Search Strategy and Study Selection}

A search strategy was created to capture all relevant publications which assessed the role of NMES for improving quadriceps weakness following total hip replacement (Table 1). Both inpatient and outpatient rehabilitation routines were included, where NMES was prescribed postoperatively to stimulate the quadriceps muscles. The search strategy generated seven results. All titles and abstracts were initially checked for relevance by two independent reviewers (LB and TW), who both hold professional positions within orthopaedic research. Four papers were omitted as they did not evaluate an NMES intervention following hip replacement surgery, and one study was removed as it investigated peripheral magnetic stimulation. Once irrelevant results were removed, the remaining articles underwent a full-text appraisal to ensure that the studies were of good methodological quality, that their findings were significant, that they were evaluating a NMES device and that they were examining the effectiveness of the device to treat quadriceps weakness or impairment. Study design was assessed using the PICO (Patient, Intervention, Comparison and Outcome) framework [34] to ensure the study was relevant. A flow diagram of the study identification process can be found in Fig. 1 in the Appendix.

\section{Methodological Assessment}

The PEDro (Physiotherapy Evidence Database) scale was used to grade the methodological quality of the two articles discussed within our review [35]. The 11-item scale is a validated measure used to assess randomised controlled trials [36] and is reported to have good inter-rater reliability (generalised kappa statistic of between 0.40 and 0.75 ) [37]. Each study was scored out of ten by two independent authors; with a score of 6 as the threshold for a high-quality study (item one on the scale indicates external validity). The PEDro scale scores ten items: random allocation, concealed allocation, similarity at baseline, subject blinding,

Table 1 Search strategy

Search strategy

1. ("Arthroplasty, Replacement, Hip") OR ("Hip Prosthesis") OR (hip*) (arthroplast* OR prosthes* OR replace*) OR "THA" OR

"THR" OR (total hip replacement)

2. AND Electric OR Electrical OR functional OR neuromuscular 3. AND Stimulation

4. AND (Muscle, Quadriceps) OR (Quadriceps Muscle, Muscles) OR "Quadriceps Femoris" OR "Vastus Medialis" OR "Vastus Intermedius" OR "Rectus Femoris" OR "Vastus Lateralis" therapist blinding, assessor binding, greater than $85 \%$ followup for at least one key outcome, intention-to-treat analysis, between-group statistical comparison for at least one key outcome and point and variability measures for at least one key outcome [36]. Quality assessment was not a factor for inclusion or exclusion within the review but was instead utilised to facilitate interpretation of findings.

\section{Summary of Current Evidence}

The search yielded seven results, two of which were relevant for inclusion within this review. A summary of the characteristics of these two studies is presented in Table 2. One study has investigated the effects of unilateral NMES on the operated side following hip replacement compared to standard home-based rehabilitation or unilateral resistance training of the operated side [32]. The patients received $1 \mathrm{~h}$ of stimulation a day for 12 weeks to the quadriceps muscles, at a pulse rate of $40 \mathrm{~Hz}$, a pulse width of $250 \mu$ s and each stimulation package lasted for $10 \mathrm{~s}$, followed by $20 \mathrm{~s}$ of rest. During the first and last $2 \mathrm{~s}$ of stimulation, the amplitude increased and decreased gradually respectively. Mean \pm standard error length of stay was shorter for the resistance training group $(10.0 \pm 2.4$ days $)$ than the electrical stimulation group ( $12 \pm 2.8$ days) and the standard rehabilitation group $(16.0 \pm 7.2$ days $)(p<.05)$. Resistance training included daily knee extension exercises $(3 \times 10$ repetitions) in the seated position with sandbags strapped to the ankle during hospitalisation. As soon as possible, training was performed on adjustable leg press and knee-extension machines. The protocol included a 10-min warm-up on a stationary bike, seated knee extensions and leg presses performed in the supine position. Training intensity was progressively increased in intensity from 20 repetition maximum (RM) $(\sim 50 \%$ of $1 \mathrm{RM})$ the first week to $15 \mathrm{RM}(\sim 65 \%$ of $1 \mathrm{RM})$ during weeks two to four to 12 $\mathrm{RM}(\sim 70 \%$ of $1 \mathrm{RM})$ during weeks five to six and finally to 8 $\mathrm{RM}(\sim 80 \%$ of $1 \mathrm{RM})$ the last 6 weeks.

Resistance training increased maximal gait speed by $30 \%$ $(p<.001)$, stair climbing performance by $28 \%(p<.005)$ and sit-to-stand score by $30 \%(p<.001)$. Electrical stimulation increased maximal gait speed by $19 \%(p<.05)$, stair climbing performance by $21 \%(p<.001)$ and sit-to-stand score by $21 \%$ $(p<.001)$. No improvements were seen in the group receiving standard, home-based rehabilitation. Quadriceps muscle cross-sectional area of the operated leg decreased by $13 \%$ at 5 weeks post-surgery in the standard care group $(p<.05)$ and remained $9 \%$ below baseline values at 12 weeks following surgery $(p<.05)$. In the resistance training group, crosssectional area of the operated leg was unaltered at 5 weeks post-surgery and increased by $12 \%$ from baseline values to 12 weeks following surgery $(p<.05)$. In the electrical stimulation group, cross-sectional area of the operated side decreased by $4 \%$ at 5 weeks following surgery $(p<.05)$ and 


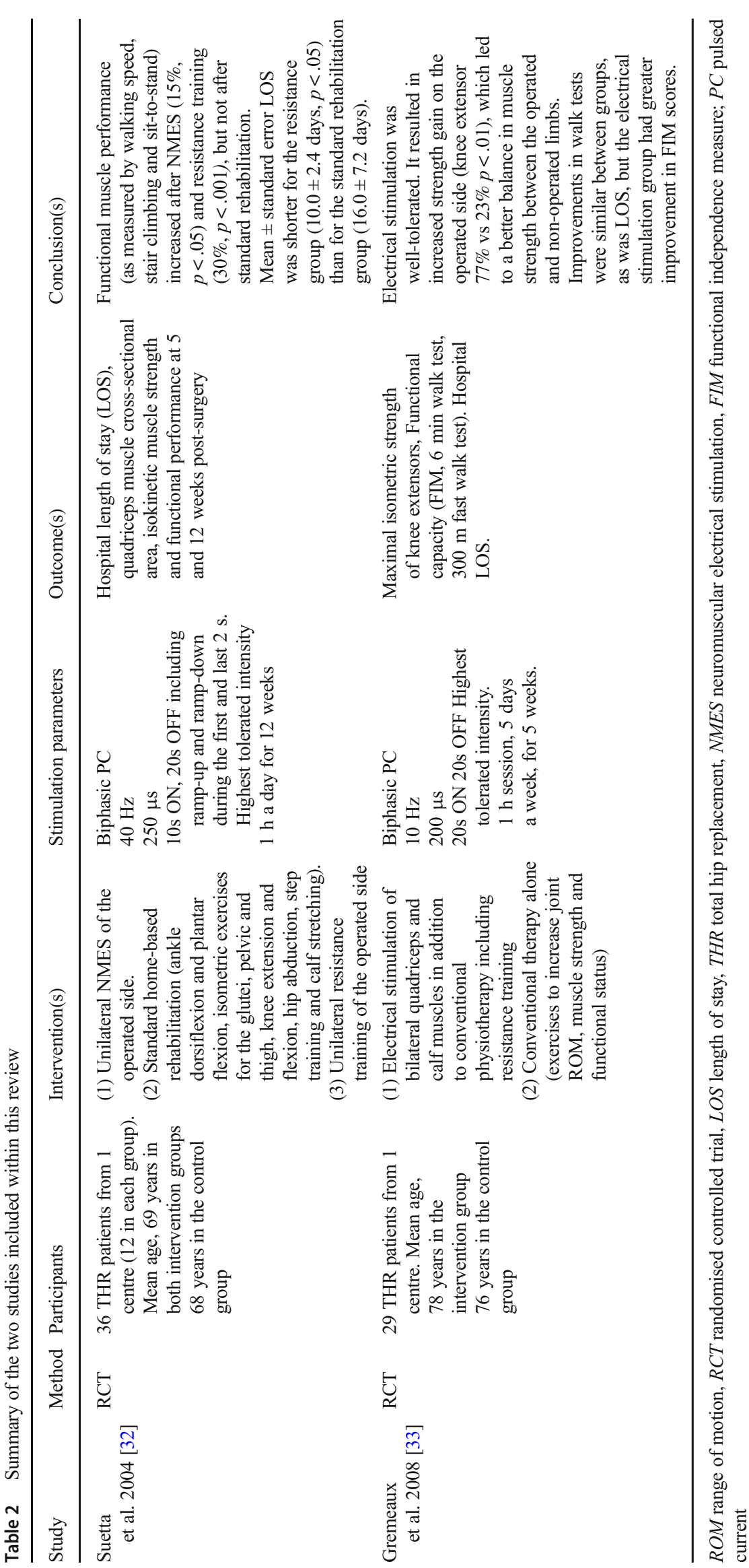


increased by $7 \%$ from 5 weeks to 12 weeks following surgery $(p<.05)$. The non-operated side was unaffected in all three groups. Peak torque improved on the operated leg for the resistance training group by $28 \%$ at $60 \% \mathrm{~s}(p<.001)$ and $22 \%$ at $180 \%$ s $(p<.05)$ at 12 weeks following surgery, but there were no changes on either leg at any time for the electrical stimulation and standard care groups [32].

Electrical stimulation in addition to physiotherapy has been compared to standard care physiotherapy alone following hip replacement surgery in one study [33]. Stimulation was applied to the quadriceps and calf muscles bilaterally using two portable dual-channel stimulators. Each delivered a 10-Hz biphasic current, with a pulse width of $200 \mu$ s and each cycle was on and off alternatively for $20 \mathrm{~s}$. As the rehabilitation intervention progressed, the stimulation intensity applied to each muscle was increased to the maximum value tolerated by the patients. The stimulation occurred for a 1-h session, 5 days a week for 5 weeks in addition to $2 \mathrm{~h}$ of physiotherapy. Low-frequency electrical muscle stimulation of the quadriceps, in addition to conventional physiotherapy, in elderly subjects (mean age 78 years) was well tolerated and led to a significant increase in muscle strength in the operated limb. There was a significant increase in maximal isometric strength of the knee extensors on the operated $(77 \%$ increase $(p<.01))$ and non-operated $(15 \%$ increase $(p<.05))$ sides in the electrical stimulation group, and in the operated limb of the control group $(23 \%$ increase $(p<.05)$. The improvement in strength was significantly greater for the electrical stimulation group than the control group for the operated limb $(p<.05)$ and was also greater for the non-operated leg (15\% vs $8 \%$ ) but did not reach statistical significance. The electrical stimulation group demonstrated significant improvements from pre-surgery to post-surgery in functional status and degree of independence (as measured by the FIM instrument) 45 days after the intervention was initiated (21.8\% increase) but the control group did not $(16 \%$ increase) $(p<.05)$. Although there were no significant differences in walking speed or length of stay between the electrical stimulation and control groups, the stimulation was advocated as a safe and effective adjunct therapy to standard care for hip replacement patients.

\section{Discussion}

Regaining muscle strength is important for postoperative function following total hip replacement [37] and as hip muscle strength and leg press power decrease substantially in the first week after surgery [20]; early rehabilitation is of significant importance. Early mobilisation, also termed early ambulation, is a well-documented component of enhanced recovery programmes that can prevent early postoperative complications such as venous thromboembolism [38] and respiratory infection [39]. Despite the well-established benefits of early mobilisation post-surgery, the role of the physiotherapist postdischarge is not fully understood and there is currently no agreed evidence-base for exercise-based physical rehabilitation to enhance postoperative recovery from total hip replacement surgery [9]. As advances in technology improve the standard and availability of assistive devices to support rehabilitation, it is possible that NMES may have a role in postoperative rehabilitation. It is acknowledged that NMES is not superior to traditional exercise for restoring muscular strength; however, it can be applied in the early phase of rehabilitation when voluntary contractions are not feasible and may enable high exercise volume, with little effort, at a time point where muscle inhibition is most prevalent [9॰].

\section{Current Evidence}

Due to the limited available studies, the small sample sizes and the differences in protocol design and patient demographics, it is not possible to offer best practice recommendations for clinical rehabilitation. NMES is reported to improve length of stay, gait speed, stair climbing performance, sit-tostand scores and reduction of quadriceps muscles crosssectional area in one study [32]. Similar results were seen with resistance training, but not with standard home-based rehabilitation [32]. Conversely, Gremeaux et al. [33] report no significant effect of NMES on length of stay or gait speed, but instead benefits to knee extensor strength of the operated side, functional status and independence. However, the participants are not necessarily comparable between these studies due to differences in protocol design and patient demographics, such as age. Both studies highlight the benefits of utilising NMES as an adjunct treatment to standard physiotherapy, although Suetta et al. [32] advocate postoperative resistance training to have a greater effect. Due to the small number of participants, conclusions must be drawn with caution.

The methodological qualities of the two studies were graded as "high quality" in accordance with the PEDro scale (Table 4 in the Appendix) [35]. Both studies were deducted points due to failure to blind subjects; however, it is acknowledged that blinding in trials using electrical stimulation is not always feasible [33]. The study by Gremeaux et al. [33] was deduced a further two points as it was not clear whether the therapists or outcome assessors were blinded. The study conducted by Suetta et al. [32] was deducted a point for item 8 (measures of at least one key outcome obtained from more than $85 \%$ of the subjects initially allocated to groups) as only 30 of the $36(83 \%)$ originally enrolled completed the study. Reasons for withdrawal were illness (2), personal problems (2) and dissatisfaction with the randomisation outcome (2). Both studies report limitations due to small sample sizes, and in addition, Gremeaux et al. [33] acknowledge their inability to standardise the exercise intensity of their rehabilitation programme, limitations to their walk tests and the absence of a true placebo group. 


\section{Neuromuscular Electrical Stimulation in Knee Replacement Surgery}

Despite the paucity of evidence for electrical stimulation in patients undergoing hip replacement, rehabilitation programmes that combine high-intensity muscle contractions in addition to NMES treatment to facilitate activation are reported to attenuate the loss of quadriceps strength following total knee replacement $[31,40]$. Electrical stimulation is reported to provide a sufficient training dose for patients lacking adequate volitional quadriceps activation by engaging neurophysiological mechanisms which facilitate strength gains and create a physical stress to the quadriceps' neuromuscular system [40]. Major muscles functioning around the hip and knee are reported to show a substantial loss of strength and mass in both patients with osteoarthritis of the hip [41] and of the knee [42]. Similarly, early postoperative strength losses and decreased functional capacity remain both post-knee replacement [43] and post-hip replacement [44] and deficits in quadriceps muscle strength have been highlighted following both procedures [21, 45]. As similarities exist in atrophic musculature following hip and knee replacement surgery, these findings are important to help inform future research.

\section{Conclusions}

Although it is not possible to offer best practice recommendations for clinical rehabilitation, the promising approach of NMES following total hip replacement requires further investigation. Combining electrical stimu- lation with standard physiotherapy care may help to attenuate the reductions in quadriceps strength, muscle fibre cross-sectional area and voluntary muscle activation which occur following surgery. NMES in addition to physiotherapy is advocated following total knee replacement for improving strength and function by combining low repetitions with rest periods to prevent muscle fatigue [40], however, further evidence is needed in cohorts of hip replacement patients. NMES may be particularly beneficial in the early acute stage of recovery, as use immediately post-surgery can enable a high exercise volume, with little effort, at a time point where muscle inhibition is most prevalent [9•]. Appropriate stimulation parameters are of paramount importance and should be a focus of future research.

\section{Compliance with Ethics Guidelines}

Conflict of Interest Thomas Wainwright reports personal non-related consultancy fees from Zimmer Biomet, Sky Medical Technology, The Technology Partnership, DePuy and Medtronic. He is a Director and Shareholder in Healthdecoded Ltd. He has received institutional research grants from Dorset Local Enterprise Partnership, Wessex Academic Health Science Network, OSSIM Technologies, Stryker and The Technology Partnership outside the submitted work. Ian Swain and Paul Taylor report patents on various Functional Electrical Stimulators and are shareholders in a stimulator manufacturing company, Odstock Medical Limited. None of these devices were used in any of the work described here. Louise Burgess declares no conflicts of interest relevant to this manuscript.

Human and Animal Rights and Informed Consent This article does not contain any studies with human or animal subjects performed by any of the authors.

\section{Appendix}

Table 3 Inclusion and exclusion criteria

\begin{tabular}{ll}
\hline Inclusion criteria & Exclusion criteria \\
\hline $\begin{array}{l}\text { Population } \\
\text { Elective, primary total hip replacement or revision } \\
\quad \text { total hip replacement. }\end{array}$ & Studies on animals \\
$\begin{array}{l}\text { Adults aged } 18 \text { or over. } \\
\text { All forms of fixation, surgical approaches and types } \\
\quad \text { of prosthetic bearing surfaces. }\end{array}$ & \\
Intervention & \\
Electrical stimulation & \\
Neuromuscular electrical stimulation (NMES) & \\
Functional electrical stimulation (FES) & Electromyographic biofeedback \\
Performed post-operatively & Implanted peripheral nerve electrodes \\
Either in an in-patient, outpatient or home/community & Neuromuscular monitoring \\
$\quad$ care setting. & Calf muscle pump stimulation \\
& High-voltage pulsed current \\
& Transcutaneous electrical nerve \\
& stimulation (TENS)
\end{tabular}


Table 3 (continued)

\begin{tabular}{ll}
\hline Inclusion criteria & Exclusion criteria \\
\hline $\begin{array}{l}\text { Outcome measure } \\
\text { Primary outcome: quadriceps strength Secondary } \\
\text { outcomes: physical functional impairment, } \\
\text { quality of life, activities of daily living, } \\
\text { postoperative pain. }\end{array}$ & Does not measure quadriceps strength \\
$\begin{array}{l}\text { Methodology } \\
\text { Randomised clinical trials } \\
\text { Non-randomised clinical trials of good } \\
\text { methodological quality }\end{array}$ & \\
& Review articles \\
Publication & Case studies \\
Published in English & Cross-sectional studies \\
Access to full text & Historical studies \\
\hline
\end{tabular}

Fig. 1 PRISMA flow diagram

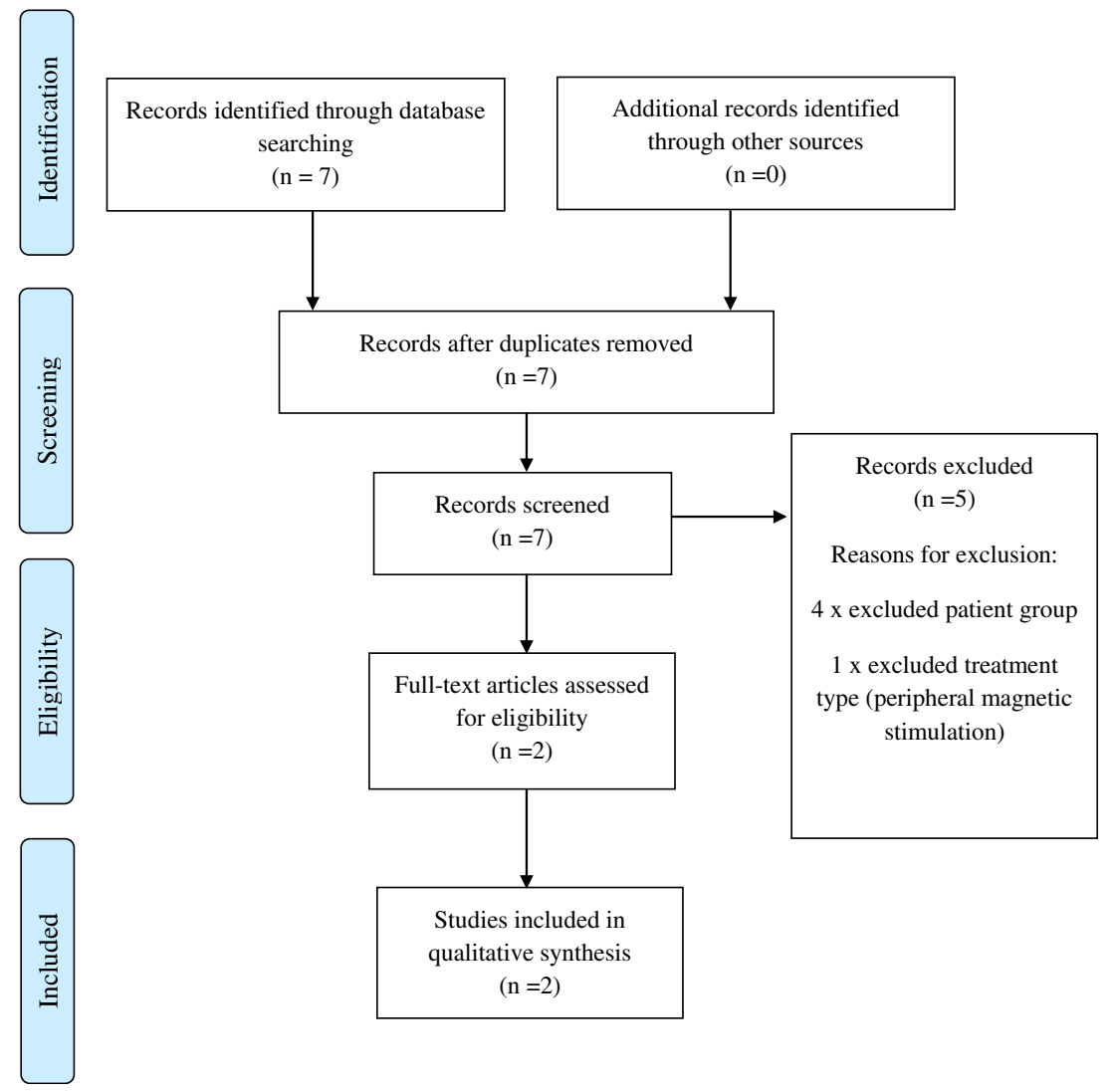

Table 4 Results from the PEDro scale

\begin{tabular}{|c|c|c|c|c|c|c|c|c|c|c|c|c|}
\hline \multirow[t]{2}{*}{ Study } & \multicolumn{11}{|c|}{ Criterion } & \multirow[t]{2}{*}{ Total } \\
\hline & 1 & 2 & 3 & 4 & 5 & 6 & 7 & 8 & 9 & 10 & 11 & \\
\hline Suetta & $\checkmark$ & $\checkmark$ & $\checkmark$ & $\checkmark$ & $X$ & $\checkmark$ & $\checkmark$ & $X$ & $\checkmark$ & $\checkmark$ & $\checkmark$ & $8 / 10$ \\
\hline Gremeaux & $\checkmark$ & $\checkmark$ & $\checkmark$ & $\checkmark$ & $\mathrm{X}$ & $X$ & $\mathrm{X}$ & $\checkmark$ & $\checkmark$ & $\checkmark$ & $\checkmark$ & $7 / 10$ \\
\hline
\end{tabular}


Open Access This article is distributed under the terms of the Creative Commons Attribution 4.0 International License (http:// creativecommons.org/licenses/by/4.0/), which permits unrestricted use, distribution, and reproduction in any medium, provided you give appropriate credit to the original author(s) and the source, provide a link to the Creative Commons license, and indicate if changes were made.

\section{References}

Papers of particular interest, published recently, have been highlighted as:

- Of importance

1. Ethgen $\mathrm{O}$, Bruyere $\mathrm{O}$, Richy $\mathrm{F}$, et al. Health-related quality of life in total hip and knee arthroplasty. A qualitative and systematic review of the literature. J Bone Joint Surg Am. 2004;86-A:963-74.

2. Beswick AD, Wylde V, Gooberman-Hill R, Blom A, Dieppe P. What proportion of patients report long-term pain after total hip or knee replacement for osteoarthritis? A systematic review of prospective studies in unselected patients. BMJ Open. 2012;2: e000435.

3. Bengtsson A, Donahue GS, Nemes S, Garellick G, Rolfson O. Consistency in patient-reported outcomes after total hip replacement. Acta Orthop. 2017;88:484-9.

4. Minnella EM, Bousquet-Dion G, Awasthi R, Scheede-Bergdahl C, Carli F. Multimodal prehabilitation improves functional capacity before and after colorectal surgery for cancer: a five-year research experience. Acta Oncol. 2017;56:295-300.

5. Miller TE, Mythen M. Successful recovery after major surgery: moving beyond length of stay. Perioper Med. 2014;8:4.

6. McNaught H, Jones T, Immins T, Wainwright TW. Patient reported importance of assistive devices in hip and knee replacement enhanced recovery after surgery (ERAS) pathways. Br J Occup Ther. 2016;79:614-9.

7. Withers TM, Lister S, Sackley C, et al. Is there a difference in physical activity levels in patients before and up to one year after unilateral total hip replacement? A systematic review and metaanalysis. Clin Rehabil. 2016;31:639-50.

8. Smith T, Withers T, Luben R, Sackley C, Jones A, MacGregor A. Changes in physical activity following total hip or knee arthroplasty: a matched case-control study from the EPIC-Norfolk cohort. Clin Rehabil. 2017;31:1548-57. This study of $\mathbf{4 0 0}$ hip or knee replacement patients highlights that following surgery, physical activity levels often do not increase and in some instances decrease.

9. Bandholm T, Wainwright TW, Kehlet H. Rehabilitation strategies for optimisation of functional recovery after major joint replacement. J Exp Orthop. 2018;5:1-4. This recent review on rehabilitation strategies emphasises the promising approach of NMES and encourages further investigation.

10. Reid KF, Naumova EN, Carabello RJ, Phillips EM, Fielding RA. Lower extremity muscle mass predicts functional performance in mobility-limited elders. J Nutr Health Aging. 2008;12(7):493-8.

11. Puthoff ML. The relationship between impairments in muscle performance, functional limitations, and disability in older adults. $\mathrm{PhD}$ thesis, University of Iowa 2006.

12. Buirs LD, Van Beers LWAH, Scholtes VAB, et al. Predictors of physical functioning after total hip arthroplasty: a systematic review. BMJ Open. 2016;6:e10725.
13. Ploutz-Snyder LL, Manini T, Ploutz-Snyder RJ, et al. Functionally relevant thresholds of quadriceps femoris strength. J Genrontol A Biol Sci Med Sci. 2002;57:144-52.

14. Mau-Moeller A, Jacksteit R, Jackszis M, Feldhege F, Weippert M, Mittelmeier W, et al. Neuromuscular function of the quadriceps muscle during isometric maximal, submaximal and submaximal fatiguing voluntary contractions in knee osteoarthrosis patients. PLoS One. 2017;12:e0176976.

15. Crockett K, Ardell K, Hermanson M, Penner A, Lanovaz J, Farthing J, et al. The relationship of knee-extensor strength and rate of torque development to sit-to stand performance in older adults. Physiother Can. 2013;65:229-35.

16. Rosendahl E, Lindelöf N, Littbrand H, Yifter-Lindgren E, LundinOlsson L, Håglin L, et al. High-intensity functional exercise program and proteinenriched energy supplement for older persons dependent in activities of daily living: a randomised controlled trial. Aust J Physiother. 2006;52:105-13.

17. American Geriatrics Society. Geriatrics Society, American Academy of Orthopaedic Surgeons panel on fall prevention. Guideline for the prevention of falls in older persons. J Am Geriatr Soc. 2001;49:664-72.

18. National Joint Registry. National Joint Registry 14th annual report. 2017. Available from: http://www.njrcentre.org.uk/. [Last accessed 02/07/2018].

19. Loureiro A, Mills PM, Barrett RS. Muscle weakness in hip osteoarthritis: a systematic review. Arthritis Care Res. 2013;65:340-52.

20. Holm B, Thorborg K, Husted H, Kehlet H, Bandholm T. Surgeryinduced changes and early recovery of hip-muscle strength, legpress power and functional performance after fast-track total hip arthroplasty: a prospective cohort study. PLoS One. 2013;8:e62109.

21. Reardon K, Galea M, Dennett X, Choong P, Byrne E. Quadriceps muscle wasting persists 5 months after total hip arthroplasty for osteoarthritis of the hip: a pilot study. Intern Med J. 2001;31:7-14.

22. Terracciano C, Celi M, Lecce D, Baldi J, Rastelli E, Lena E, et al. Differential features of muscle fiber atrophy in osteoporosis and osteoarthritis. Osteoporos Int. 2013;24:1095-100.

23. Lewek MD, Rudolph KS, Snyder-Mackler L. Quadriceps femoris muscle weakness and activation failure in patients with symptomatic knee osteoarthritis. J Orthop Res. 2004;22:110-5.

24. Watson T. Electrotherapy: evidence-based practice. 12th ed. Philadelphia: Elsevier Ltd; 2008.

25. Spector P, Laufer Y, Elboim GM, et al. Neuromuscular electrical stimulation therapy to restore quadriceps muscle function in patients after orthopaedic surgery: a novel structured approach. J Bone Joint Surg Am. 2016;98:2017-24.

26. Gibson JN, Smith K, Rennie MJ. Prevention of disuse muscle atrophy by means of electrical stimulation: maintenance of protein synthesis. Lancet. 1988;1(2):767-70.

27. Snyder-Mackler L, Delitto A, Bailey SL, Stralka SW. Strength of the quadriceps femoris muscle and functional recovery after reconstruction of the anterior cruciate ligament. A prospective, randomized clinical trial of electrical stimulation. J Bone Joint Surg Am. 1995;77:1166-73.

28. Benton LA, Baker LL, Bowman BR, et al. Functional electrical stimulation - a practical clinical guide. 2nd ed. California: The Professional Staff Association of the Rancho Los Amigos Hospital.

29. Hainaut K, Duchateau J. Neuromuscular electrical stimulation and voluntary exercise. Sports Med. 1992;14:100-13.

30. Vanderthommen M, Duchateau J. Electrical stimulation as a modality to improve performance of the neuromuscular system. Exerc Sport Sci Rev. 2007;35(4):180-5.

31. Bistolfi A, Zanovello J, Ferracini R, et al. Evaluation of the effectiveness of neuromuscular electrical stimulation after total knee arthroplasty: a meta-analysis. Am J Phys Med Rehabil. 2018;97: 123-30. This thorough review highlights the role of NMES for improving recovery from total knee replacement. 
32. Suetta C, Magnusson SP, Rosted A, Aagaard P, Jakobsen AK, Larsen LH, et al. Resistance training in the early postoperative phase reduces hospitalisation and leads to muscle hypertrophy in elderly hip surgery patients - a controlled randomised study. J Am Gertiatr Soc. 2004;52:2016-22.

33. Gremeaux V, Renault J, Pardon L, Deley G, Lepers R, Casillas JM. Low-frequency electric muscle stimulation combined with physical therapy after total hip arthroplasty for hip osteoarthritis in elderly patients: a randomized controlled trial. Arch Phys Med Rehabil. 2008;89:2265-73.

34. Huang X, Lin J, Demner-Fushman D. Evaluation of PICO as a knowledge representation for clinical questions. AMIA Annu Symp Proc. 2006;2006:359-63.

35. Physiotherapy Evidence Database. 1999. The PEDro scale. https:// www.pedro.org.au/wp-content/uploads/PEDro_scale.pdf. [Accessed 02 January 2019].

36. De Morton NA. The PEDro scale is a valid measure of the methodological quality of clinical trials: a demographic study. Aust J Physiother. 2009;55:129-33.

37. Maher CG, Sherrington C, Herbert RD, Moseley AM, Elkins M. Reliability of the PEDro scale for rating quality of randomized controlled trials. Phys Ther. 2003;83:713-21.

38. Barker RC, Marval P. Venous thromboembolism: risks and prevention. Contin Educ Anaesth Crit Care Pain. 2011;11:18-23.

39. Boden I, Skinner EH, Browning L, et al. Preoperative physiotherapy for the prevention of respiratory complications after upper ab- dominal surgery: pragmatic, double blinded, multicentre randomised controlled trial. BMJ. 2018;360:j5915.

40. Nussbaum EL, Houghton P, Anthony J. Neuromuscular electrical stimulation for treatment of muscle impairment: critical review and recommendations for clinical practice. Physiotherapy Canada Special Issue. 2017;69:1-76. This extensive review highlights the role of NMES for the treatment of muscle impairment in a wide range of patients.

41. Rasch A, Bystrom AH, Dalen N, et al. Reduced muscle radiological density, cross-sectional area and strength of major hip and knee muscles in 22 patients with hip osteoarthritis. Acta Orthop. 2007;78:505-10.

42. Mizner RL, Petterson SC, Stevens JE, Axe MJ, Snyder-Mackler L. Preoperative quadriceps strength predicts functional ability one year after total knee arthroplasty. J Rheumatol. 2005;32:1533-9.

43. Judd DL, Dennis DA, Thomas AC, Wolfe P, Dayton MR, StevensLapsley JE. Muscle strength and functional recovery during the first year after THA. Clin Orthop Relat Res. 2014;472:654-64.

44. Fukumoto Y, Ohata K, Tsukagoshi R, et al. Changes in hip and knee muscle strength in patients following total hip arthroplasty. J Jpn Phys Ther Assoc. 2013;6:22-7.

45. Mizner RL, Stevens JE, Snyder-Mackler L. Voluntary activation and decreased force production of the quadriceps femoris muscle after total knee arthroplasty. Phys Ther. 2003;83:359-65.

Publisher's Note Springer Nature remains neutral with regard to jurisdictional claims in published maps and institutional affiliations. 